Spectroscopic measurements of plasma emission light for plasma-based acceleration experiments

This content has been downloaded from IOPscience. Please scroll down to see the full text. 2016 JINST 11 C09015

(http://iopscience.iop.org/1748-0221/11/09/C09015)

View the table of contents for this issue, or go to the journal homepage for more

Download details:

IP Address: 151.100.38.9

This content was downloaded on 29/05/2017 at 12:17

Please note that terms and conditions apply.

You may also be interested in:

Experiments on the excitation of large amplitude plasma waves for ultra-relativistic particle acceleration

G Matthieussent

Nonparaxial theory of laser-driven plasma waves phase velocity in partially stripped magneto-plasma channels and wakefield acceleration

B S Sharma and Arachna Jain

A beat wave experiment in an open resonator

G Di Massa, R Fedele, G Miano et al.

First Beam Measurements of the S-Band photocathodeRadio-Frequency Gun at Tsinghua University Du Ying-Chao, Yan Li-Xin, Du Qiang et al.

Excitation of large amplitude plasma waves

Francis F Chen

Excitation of large amplitude plasma waves in a plasma filled open resonator

U De Angelis, R Fedele, G Miano et al.

Cyclotron resonance effect on Wakefield excitation and electron acceleration

Saleh T Mahmoud and A K Sharma

Relativistic effect on stimulated Raman scattering of a laser in plasma

D N Gupta, Sushila Yadav, V K Tripathi et al.

Self-modulation instability of ultra-relativistic particle bunches with finite rise times

$\mathrm{J}$ Vieira, L D Amorim, Y Fang et al. 


\section{Spectroscopic measurements of plasma emission light for plasma-based acceleration experiments}

F. Filippi, ${ }^{a, b, 1}$ M.P. Anania, ${ }^{c}$ A. Biagioni, ${ }^{c}$ E. Chiadroni, ${ }^{c}$ A. Cianchi, ${ }^{d}$ M. Ferrario, ${ }^{c}$ A. Mostacci, ${ }^{a, b}$ L. Palumbo ${ }^{a, b}$ and A. Zigler ${ }^{e}$

${ }^{a}$ Dipartimento di Scienze di Base e Applicate per l'Ingegneria (SBAI), 'Sapienza' Università di Roma, Via A. Scarpa 14-16, Roma, 00161 Italia

${ }^{b}$ INFN-Romal, Piazzale Aldo Moro, 2, Roma, 00161 Italia

${ }^{c}$ Laboratori Nazionali di Frascati, INFN,

Via E. Fermi 40, Frascati, 00044 Italia

${ }^{d}$ Dipartimento di Fisica, Università di Roma 'Tor Vergata',

Via della Ricerca Scientifica 1, Roma, 00133 Italia

${ }^{e}$ Hebrew University of Jerusalem,

Jerusalem, 91904 Israel

E-mail: francesco.filippi@roma1.infn.it

AвSTRACT: Advanced particle accelerators are based on the excitation of large amplitude plasma waves driven by either electron or laser beams. Future experiments scheduled at the SPARC_LAB test facility aim to demonstrate the acceleration of high brightness electron beams through the so-called resonant Plasma Wakefield Acceleration scheme in which a train of electron bunches (drivers) resonantly excites wakefields into a preformed hydrogen plasma; the last bunch (witness) injected at the proper accelerating phase gains energy from the wake. The quality of the accelerated beam depends strongly on plasma density and its distribution along the acceleration length. The measurements of plasma density of the order of $10^{16}-10^{17} \mathrm{~cm}^{-3}$ can be performed with spectroscopic measurements of the plasma-emitted light. The measured density distribution for hydrogen filled capillary discharge with both Balmer alpha and Balmer beta lines and shot-to-shot variation are here reported.

Keywords: Plasma diagnostics - interferometry, spectroscopy and imaging; Wake-field acceleration (laser-driven, electron-driven)

\footnotetext{
${ }^{1}$ Corresponding author.
} 


\section{Contents}

1 Introduction $\quad 1$

$\begin{array}{llr}1.1 & \text { Experimental setup } & 2\end{array}$

2 Plasma density measurements $\quad 3$

2.1 Comparison between Balmer alfa and Balmer beta results 3

$\begin{array}{lll}2.2 & \text { Shot-to-shot variation } & 5\end{array}$

3 Conclusions 6

\section{Introduction}

It is well known in the scientific community that plasma can sustain high electric fields which have been used in recent years for accelerating charged particles [1]. Self-injection plasma-based accelerators, in which the accelerated electrons are trapped from the electron plasma itself, have proved to reach high energy in relatively short distances [2], but the large energy spread of these beams makes them not suitable for driving radiation sources or for particle colliders. External injection techniques $[3,4]$ on the opposite have the aim to accelerate pre-generated high brightness electron beams (HBEBs) with the high gradients allowed by the plasma preserving the quality of the beams. The external injection experiments that are ongoing at SPARC_LAB [5] will study the possibility to accelerate an electron beam (witness) of small energy spread $(<0.1 \%)$ and low emittance ( $\sim 1 \mathrm{~mm} \mathrm{mrad})$ using a pre-formed hydrogen plasma in which, respectively, a train of electrons or a laser pulse (drivers) has excited wakefields. Since the excitation of the wakefields is a resonant process, the acceleration mechanism depends on the plasma frequency, which is related with the electron plasma density by the well known equation $\omega_{p}=\sqrt{\frac{n_{e} e^{2}}{\epsilon_{c} m_{e}}}$ (in SI units). The proper engineering of the longitudinal plasma density is crucial to inject the accelerating beam at the proper delay respect to the driver(s), avoiding defocusing or decelerating fields. Transverse plasma distribution has already been investigated both analytically [6] and experimentally [7] in the past, showing that the electron density configuration of a hydrogen-filled cylindrical capillary is approximately flat around the axis. This makes these kind of capillary suitable for electron driven plasma wakefield acceleration experiments since it allows the beams to pass through a transversally homogeneous plasma channel during the acceleration process. Moreover, in the shotto-shot variation of the plasma density the uniformity of the density within several percent is a general requirement.

In this paper, we show the setup for the spectroscopic investigations of the Stark broadening for hydrogen plasma. This setup allows for a single shot measurement of the longitudinal plasma density with the temporal resolution of the gated camera (tens of nanoseconds). With this device it has been possible to investigate the plasma electron density profile during a hydrogen-filled capillary discharge and its repeatability. Some measurements of the plasma evolution for gas-filled capillary discharge are shown below in the text. 


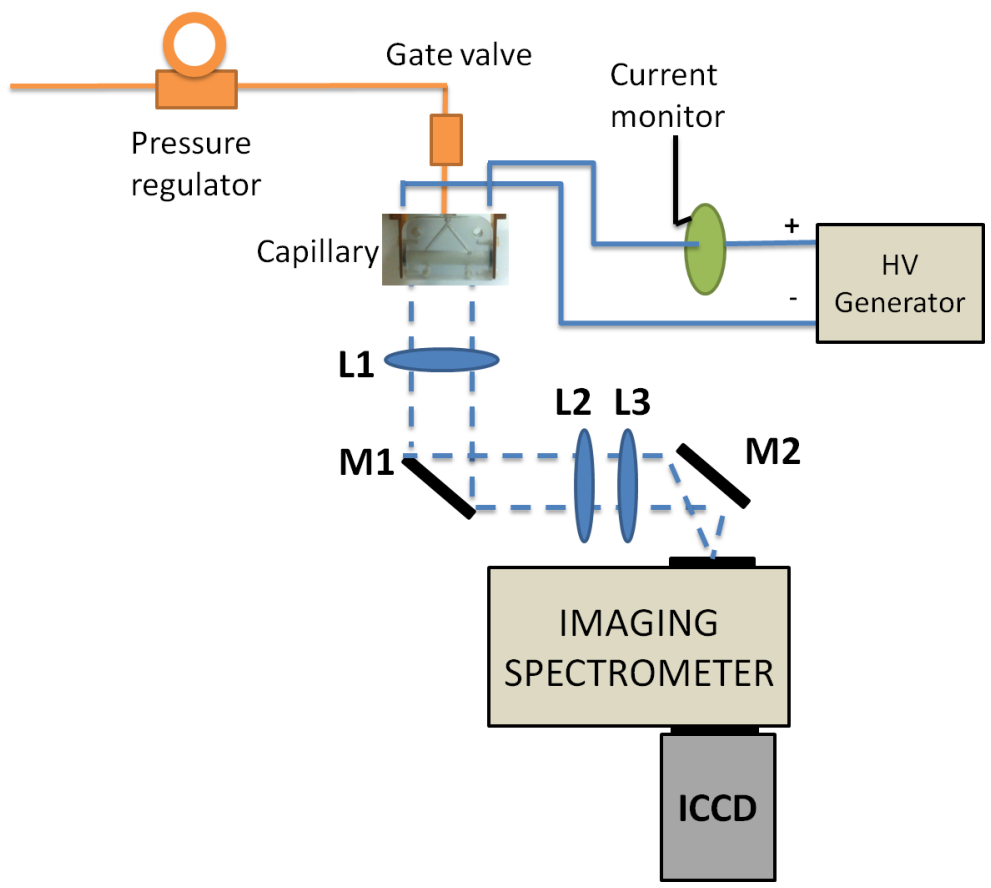

Figure 1. Experimental setup used for the plasma production in gas filled capillary and Stark broadening measurements of the plasma density. The inlet pressure of the hydrogen is regulated by a pressure regulator placed outside the vacuum chamber. A high speed solenoid valve lead the gas to flow in. The high voltage (HV) generator applies a voltage of $20 \mathrm{kV}$ between the two ends of the capillary. The light emitted by the plasma inside the capillary is collected by a system of lenses and it is sent into the spectrometer slit. A fast-gated intensified Andor camera was used as a detector for the spectrometer.

\subsection{Experimental setup}

Experiments were performed at the SPARC_LAB test facility (INFN National Laboratory in Frascati).

The layout of the experiment is shown in figure 1. A sapphire capillary of circular cross section of $1 \mathrm{~mm}$ diameter is fed by two gas inlets of $0.5 \mathrm{~mm}$ diameter placed at $7.5 \mathrm{~mm}$ from the respective end. The hydrogen is produced by the electrolysis of water by the NM Plus Hydrogen Generator furnished by Linde. The inlet pressure of the gas is imposed outside the vacuum chamber at more than $1 \mathrm{~m}$ from the capillary and was imposed by a regulator to $100 \mathrm{mbar}$. The inlet pressure was not varied during the measurements reported in this article. A high speed solenoid valve is placed at $5 \mathrm{~cm}$ from the capillary. The valve is opened for $3 \mathrm{~ms}$ then the discharge happens few microseconds after its closure. The short time of the valve opening is dictated mainly by the pressure inside the test chamber that has been maintained below $1 \cdot 10^{-7}$ mbar before every shot.

Between the two ends of the capillary was applied a voltage of $20 \mathrm{kV}$ by a capacitor of $2 \mathrm{nF}$ charged by a discharge circuit. The current of the discharge can be monitored by a Pearson current monitor placed around one of the two wires that feeds the capillary electrodes. During the experiment, currents of $180 \mathrm{~A}$ were measured.

The light emitted by the plasma is collected by a system of lenses that produces an image of the capillary onto the entrance slit of an imaging spectrometer (SP 275 of the ARC Corporation). 
The spectrometer has an aperture ratio of $\mathrm{f} / 3.8$, focal length of $275 \mathrm{~mm}$, and it is equipped with a grating of $2400 \mathrm{~g} / \mathrm{mm}$. A fast-gated, intensified camera (Andor iSTAR 320) is used as detector for the spectrometer. We obtained the time dependence of the plasma density by scanning the relative delay between the discharge trigger and the camera gate. The spatial variation of the plasma density along the capillary was obtained by analyzing different rows of the spectral image corresponding to different points inside the capillary. This allows to measure the density distribution along the capillary in a single shot.

\section{Plasma density measurements}

Hydrogen atoms, when excited, emit light at different wavelengths in the visible range, usually referred to as Balmer spectral lines. The analysis of the line broadening allows to reconstruct the plasma electron density around the emitting atoms due to the Stark effect. Balmer alfa is the strongest of the Balmer lines, but it is also more sensitive to the plasma temperature effects and it leads often to an overestimation of the plasma density [8]. On the other hand, Balmer beta shows a greater Stark width even if its strength is weaker. In this article, we have analyzed both the Balmer alpha and the Balmer beta lines emitted by the hydrogen excited in the gas-filled capillary discharge.

Many formulas are available elsewhere for the estimation of the electron density from the Stark broadening. For the Balmer alpha line, authors like Gigosos [9] refused to give a simple analytical formula to convert the full width at half maximum (FWHM) of the emitted line to electron density, while other authors like Griem [10], although giving an analytical formula, did not take into account the ion dynamics. For that reason we decided to infer our formula from the experimental data given by Griem [11], which are in the temperature range of our interest (around $1-4 \mathrm{eV}$ ), as we already done in previous works [12]. The analytical formula we have used is the following:

$$
\Delta \lambda[\mathrm{nm}]=\left[n_{e}\left[10^{18} \mathrm{~cm}^{-3}\right]\right]^{2 / 3} \cdot 5.34 .
$$

We compared this formula with the one given by Griem in 1979 [10], the mismatch between these formulas for densities between $5 \cdot 10^{15}$ and $1 \cdot 10^{18} \mathrm{~cm}^{-3}$ is lower than $3 \%$. For the Balmer beta line, we used the analytical formula given by Gigosos and Cardeñoso [9] obtained with the so-called Gig-Card model, which takes into account the ion dynamics and gives more accurate results

$$
\Delta \lambda[\mathrm{nm}]=\left[n_{e}\left[10^{18} \mathrm{~cm}^{-3}\right]\right]^{1.4680} \cdot 4.8 .
$$

Due to the low plasma temperature, the Doppler broadening acting on the Balmer lines is much smaller than the Stark, so we could interpolate the lines with a Lorentzian function.

\subsection{Comparison between Balmer alfa and Balmer beta results}

The light emitted by the hydrogen is collected and sent to the spectrometer by an imaging system of three lenses and two mirrors. The capillary is imaged on the entrance slit of the imaging spectrometer, then with the gated camera it is possible to analyze the image of the capillary spectrally dispersed by the spectrometer. By varying the delay between the camera trigger and the discharge trigger it was possible to scan the temporal evolution of the plasma as well as its spatial variation. The measurements exposed in this article has been performed by varying the delay with steps of $100 \mathrm{~ns}$ and with $100 \mathrm{~ns}$ of camera gating time. Density profiles obtained with both Balmer alpha and Balmer beta line for 400, 800 and $1000 \mathrm{~ns}$ delay are presented in figure 2 . 


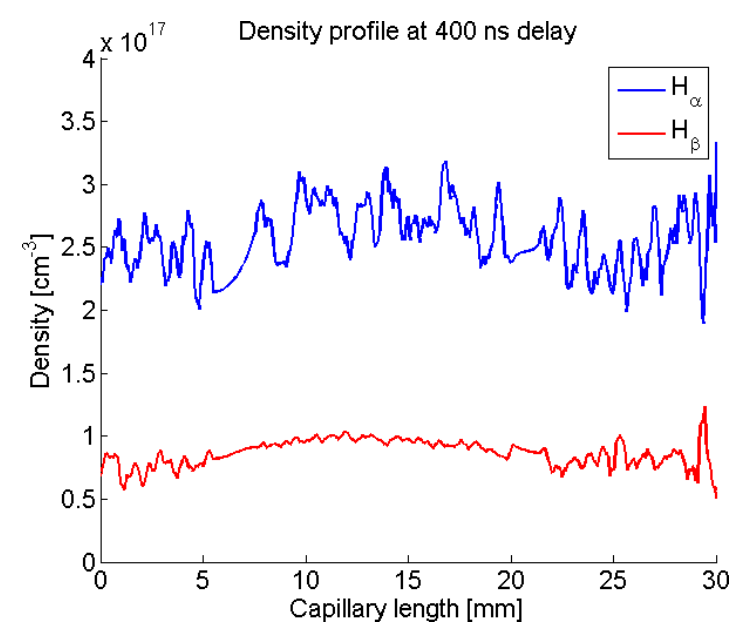

(a) $400 \mathrm{~ns}$.

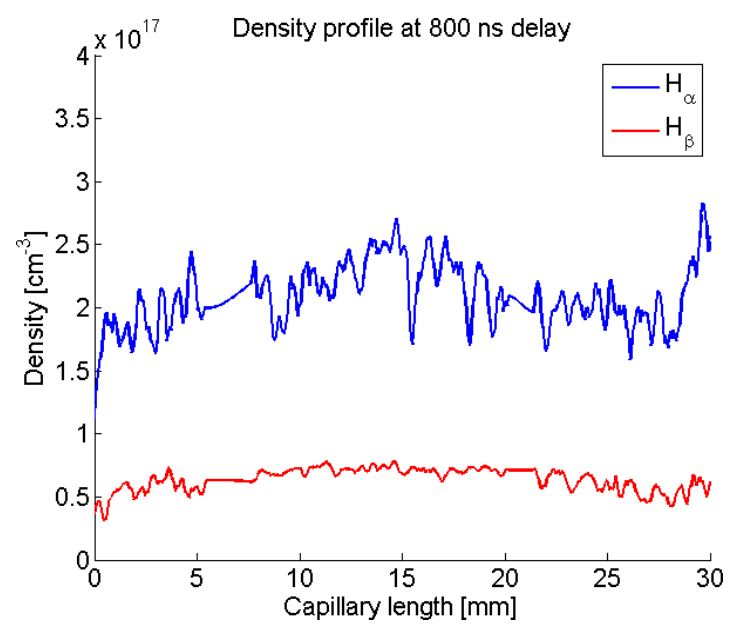

(b) $800 \mathrm{~ns}$.

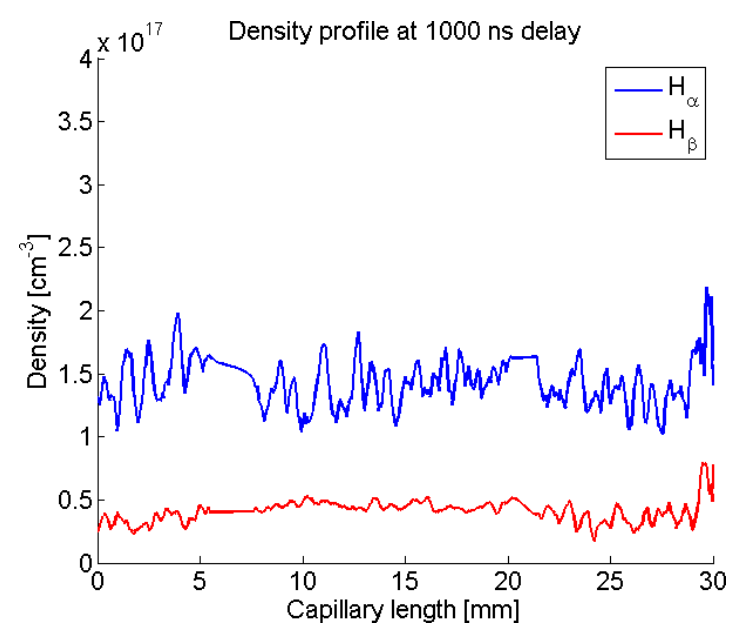

(c) $1000 \mathrm{~ns}$.

Figure 2. Density variation along the capillary at different delays from the discharge trigger, namely $400 \mathrm{~ns}$, $800 \mathrm{~ns}$ and $1000 \mathrm{~ns}$. The density measured with the Balmer alpha value is almost 3 times higher than the one measured with the Balmer beta line. 
The density measured with the Balmer alpha line is higher than the one measured with the Balmer beta of about 3 times. The deviation between these two values is believed to come from the fact that Balmer alpha is susceptible to strong self-absorption as already noted in previous works $[8,13]$. The self-absorption of the central line of the emitted Balmer alpha is relatively higher than the one of the Balmer beta line, and its effect is quite severe for pure composition plasma [14] like the one we have used for our experiment. The higher self-absorption of the central line makes the measured Balmer alpha broadened, then the fit of the line is affected by the absorption and the measured density is often overestimated. Balmer beta is still affected by this phenomenon, and this may lead to a weaker over estimation of the density. Some authors have compared the results obtained with the Stark broadening analysis with the interferometric technique [13]. It has been showed that the analysis of the alpha and beta Balmer lines in the density range of our interest give higher density values compared with the values measured with the interferometry, but the Balmer beta results, reasonably due to the lower self-absorption, are closer to the interferometric values within few tens of percentage, confirming the validity of this method.

In our measurements, as can be seen in figure 2, the plasma density increases until $400 \mathrm{~ns}$, then it reaches its maximum and starts to decrease at almost $600 \mathrm{~ns}$ after the discharge trigger. The spatial distribution of the plasma density shows a central maximum that decreases close to the electrodes. This maximum is less evident after some hundreds of nanoseconds. The same qualitative trend can be observed in both the measurements performed with both the lines.

\subsection{Shot-to-shot variation}

By acquiring more than one shot with the same delay time it was possible to analyze the shot-to-shot variation of the density at the different instants of the discharge. For this analysis we imaged only the central part of the capillary between the 7 th and the 21 st millimeter. For every delay time we have evaluated the mean density profile and the maximum error (expressed in percentage) for every point along the imaged part of the capillary averaged on 5 shots. In figure 3 the mean error values for every delay is presented for both Balmer alpha and Balmer beta line.

Both curves show almost the same values except in the shots taken at $200 \mathrm{~ns}$ and $1200 \mathrm{~ns}$, where the error of the Balmer beta line is higher than the one of the Balmer alpha. This may be caused by the stronger signal of the Balmer alpha, which make easier the detection of the line profile even for lower densities. The average error for both the lines is higher when the density is lower, i.e. when the process of the discharge is still forming and during the recombination, while it is lower when the density is higher. This may be caused by the lower signal strength caused by the lower densities but also by a real density variation caused by the ionization and recombination process. The mean error value between $300 \mathrm{~ns}$ and $800 \mathrm{~ns}$ after the discharge trigger for both the lines is of the order of $10 \%$ and in any case lower than $15 \%$. 


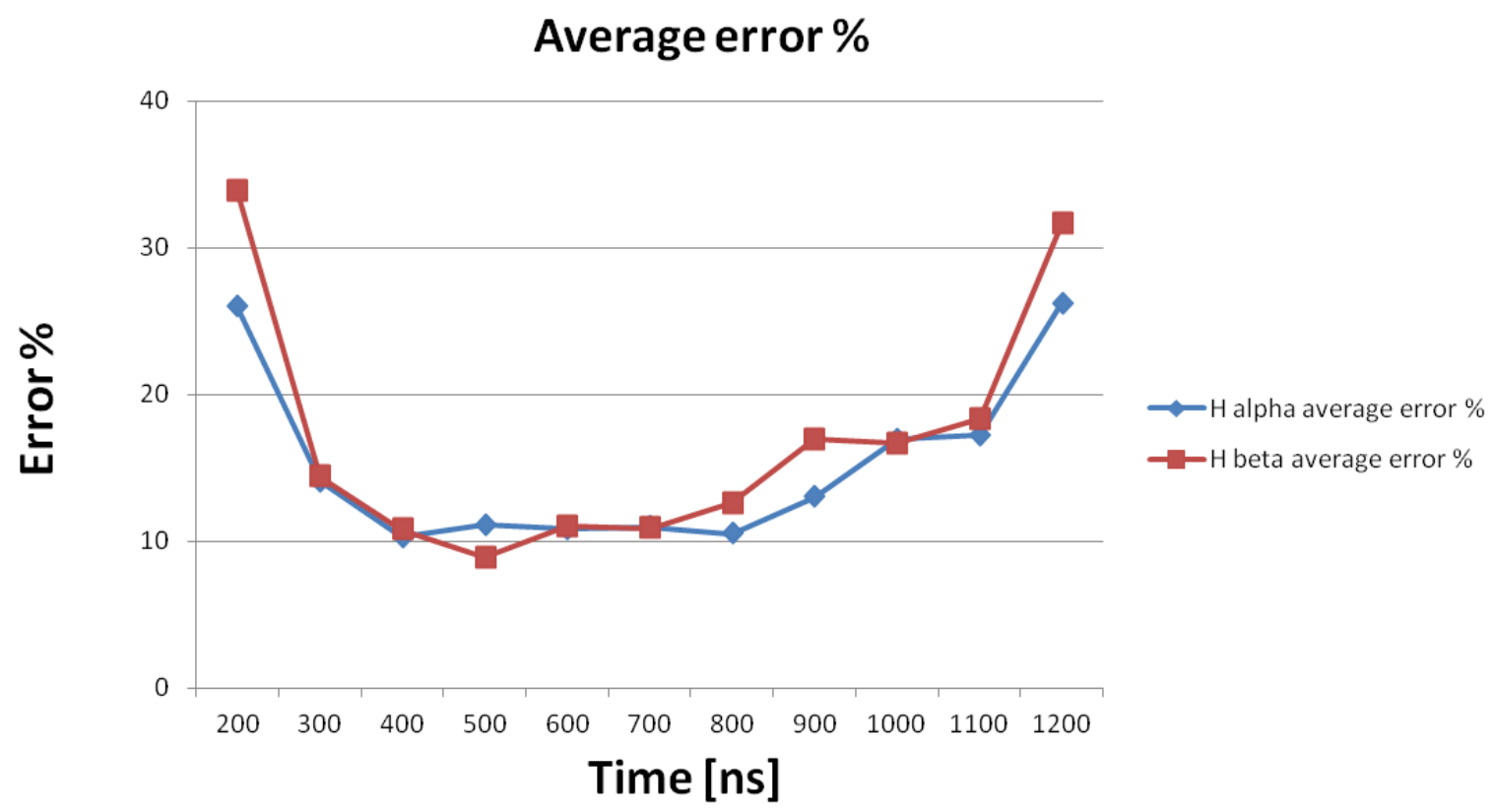

Figure 3. Error values averaged over $14 \mathrm{~mm}$ in the middle of the capillary for different time delay with respect to the discharge trigger. The error has been calculated by analyzing 5 images per each shot.

\section{Conclusions}

We have investigated the electron density variation in a hydrogen filled capillary discharge by using spectroscopic analysis of the self emitted light of the hydrogen. The Stark broadening of both the Balmer beta and Balmer alpha were analyzed. The plasma density temporal evolution has been analyzed and the density profiles for 400,800 and 1000 ns delay has been compared. The Balmer beta line results show a density value 3 times lower than the Balmer alpha, this may be caused by the re-absorption of the line and the ion dynamic effects that lead to an overestimation of the plasma density for the Bamer alpha line.

The shot-to-shot variation of the plasma density inside the capillary for different delay values has also been analyzed. The density is more stable around its mean value between $300 \mathrm{~ns}$ and $800 \mathrm{~ns}$ delay, while is higher when the density is lower. We argued that this may be caused by the lower signal strength of the lines and by a physical density variation caused by the uncertainty of the ionization and recombination process.

\section{Acknowledgments}

Authors would like to acknowledge V. Lollo and S. Bini for technical support.

This work has been supported by the EU Commission in the Seventh Framework Program, Grant Agreement 312453-EuCARD-2 and the Italian Minister of Research in the framework of FIRB — Fondo per gli Investimenti della Ricerca di Base, Project n. RBFR12NK5K.

\section{References}

[1] E. Esarey, C.B. Schroeder and W.P. Leemans, Physics of laser-driven plasma-based electron accelerators, Rev. Mod. Phys. 81 (2009) 1229.

[2] W.P. Leemans et al., GeV electron beams from a centimetre-scale accelerator, Nat. Phys. 2 (2006) 696. 
[3] M. Litos et al., High-efficiency acceleration of an electron beam in a plasma wakefield accelerator, Nature 515 (2014) 92.

[4] J.B. Rosenzweig et al., Experimental Observation of Plasma Wake Field Acceleration, Phys. Rev. Lett. 61 (1988) 98.

[5] M. Ferrario et al., SPARC_LAB present and future, Nucl. Instrum. Meth. B 309 (2013) 183.

[6] N.A. Bobrova et al., Simulations of a hydrogen-filled capillary discharge waveguide, Phys. Rev. $\mathbf{E} 65$ (2002) 016407.

[7] D.J. Spence and S.M. Hooker, Investigation of a hydrogen plasma waveguide, Phys. Rev. $\mathbf{E} 63$ (2000) 015401(R).

[8] M.A. Gigosos and V. Cardeñoso, New plasma diagnosis tables of hydrogen Stark broadening including ion dynamics, J. Phys. B 29 (1996) 4795.

[9] M.A. Gigosos and V. Cardeñoso, Computer simulated Balmer-alpha, -beta and -gamma Stark line profiles for non-equilibrium plasmas diagnostics, Spectrochim. Acta B 58 (2003) 1489.

[10] H. Griem, Spectral line broadening by plasmas, Pure Appl. Phys. 39 (1974) 1.

[11] H. Griem, J. Halenka and W. Olchawa, Comparison of hydrogen Balmer-alpha Stark profiles measured at high electron densities with theoretical results, J. Phys. B 39 (2005) 975.

[12] F. Filippi et al., Plasma density characterization at SPARC_LAB through Stark broadening of Hydrogen spectral lines, Nucl. Instrum. Meth. A 829 (2016) 326.

[13] D.G. Jang, M.S. Kim, I.H. Nam, H.S. Uhm and H. Suk, Density evolution measurement of hydrogen plasma in capillary discharge by spectroscopy and interferometry methods, Appl. Phys. Lett. 99 (2011) 141502.

[14] J. Torres, J. Jonkers, M.J. van de Sande, J.J.A.M. van der Mullen, A. Gamero and A. Sola, An easy way to determine simultaneously the electron density and temperature in high-pressure plasmas by using Stark broadening, J. Phys. D 13 (2003) L55. 